\title{
Trichobezoar: An Unusual Cause of Gastric Perforation
}

\author{
EL Ghazi M. , Zaitouni K, Boutakioute B, Idrissi Ouali M, Cherif Idrissi Ganouni N
}

Department of Radiology Arrazi Hospital, Mohamed VI University Hospital, Cadi Ayyad University, Marrakech, Morocco

DOI: $10.36347 /$ sjmcr.2020.v08i01.016

| Received: 27.12.2019 | Accepted: 04.01.2020 | Published: 18.01.2020

*Corresponding author: Mohamed EL GHAZI

\section{Abstract}

Introduction: Trichobezoar is a Greek word trich, which means hair. Bezoars are collections of indigestible material that accumulate into the gastro-intestinal tract and are most often located in the stomach. Trichobezoars are often associated with psychiatric illness like trichotillomania and trichophagia usually occurs in young and adolescent females. In this case study, we showed the interest of imaging in the the diagnosis of this entity. Case presentation: A 38 years old woman, 5 days post-partum presented in the emergency department with complete occlusive syndrome evolving for 3 days. On examination patient look irritable, abdomen was tense and sensitive on palpation. Ultrasound shows collection of free fluid in the peritoneal cavity. A complement by CT scan demonstrated a large gastric intraluminal heterogeneous mass that remined bezoars. On emergency, an exploratory laparotomy was performed and bulky gastric trichobezoar was removed. Conclusion: Gastric perforation is a rare complication of gastric trichobezoar formation. Without a history of trichotillomania or trichophagia, one would not readily include this entity on their differential diagnosis of an acute abdomen. Once diagnosed, often incidentally with the help of radiologic imaging.

Keywords: trichobezoar; trichotillomania; trichophagia; gastric perforation.

Copyright @ 2020: This is an open-access article distributed under the terms of the Creative Commons Attribution license which permits unrestricted use, distribution, and reproduction in any medium for non-commercial use (NonCommercial, or CC-BY-NC) provided the original author and source are credited.

\section{INTRODUCTION}

Trichobezoar is a Greek word trich, which means hair. Bezoars are collections of indigestible material that accumulate into the gastro intestinal tract and are most often located in the stomach. Trichobezoars are often associated with psychiatric illness like trichotillomania and trichophagia usually occurs in young and adolescent females. Gastric trichobezoar is the most common variety of bezoar found in the stomach. The common complications reported over the years, include gastric mucosal erosion, ulceration, and perforation of the stomach. In this case study, we present the 38 years old female who suffer from Trichobezoar with Gastric Perforation which is rare in nature.

\section{Case Presentation}

We report the case of a 38 years old female patient, 5 days post-partum. Presented in the emergency department with complete occlusive syndrome evolving for 3 days. On examination patient look irritable, abdomen was tense and sensitive on palpation. Ultrasound shows collection of free fluid in the peritoneal cavity. A CT of the abdomen revealed pneumoperitoine and a potentially perforated stomach, secondary to a heterogeneous mass completely filling and distending the gastric cavity( Figure 1 and 2).

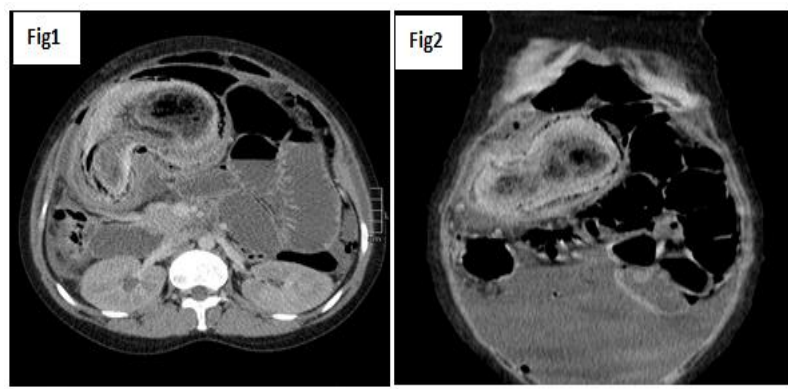

Fig-1 and 2: Axial and coronal CT images shows the large gastric intraluminal heterogeneous mass. Pneumoperitoneum is also seen

The patient underwent an emergent exploratory laparotomy, and the lesser curvature of the stomach was noted to be visibly perforated and leaking gastric fluid (Figure3). An incision was made along the posterior aspect of the stomach and a trichobezoar encompassing the entirety of the patient's stomach was visualized. The mass measured 18.5 x 11 x 7 centimeters and was removed (Figure4). The gastrostomy was repaired primarily and the gastric perforation was sutured. 


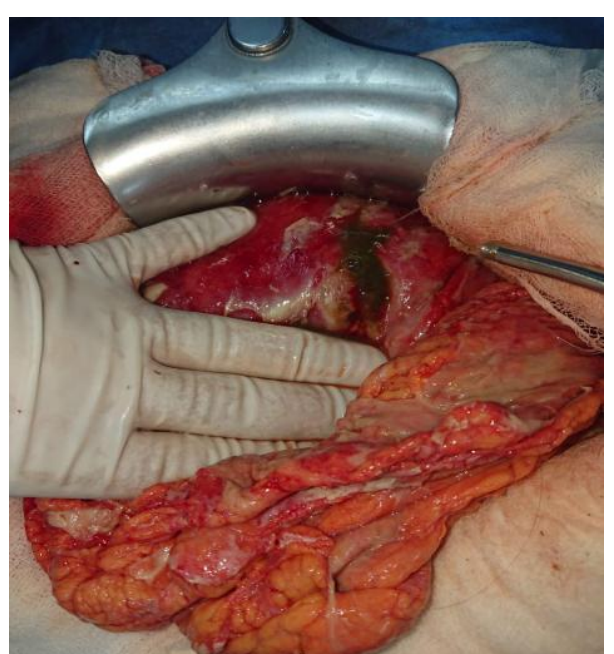

Fig-3: Perforation in lesser curvature of the stomach leaking gastric fluid.

Postoperatively her recovery was punctuated only by a right-sided pleural effusion which spontaneously. The patient was discharged home on postoperative day 14 after receiving intravenous antibiotic Psychiatric treatment was started.

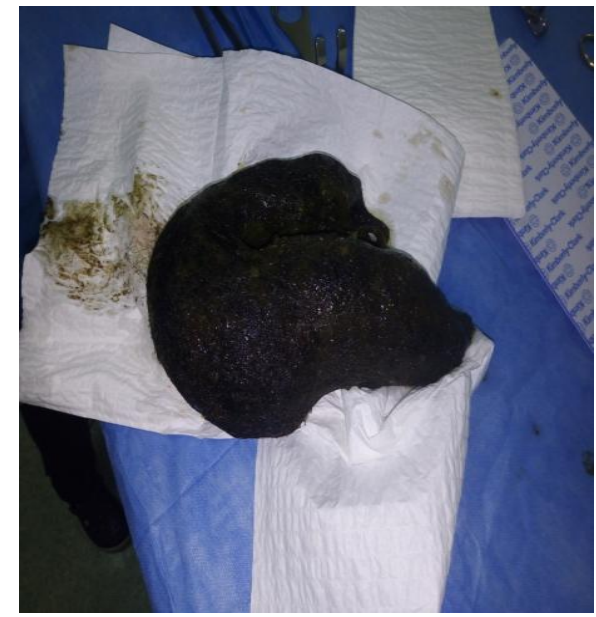

Fig-4: Surgical specimen shows the resected gastric trichobezoar

\section{DISCUSSION}

Bezoar is an uncommon but potentially serious cause of abdominal pain. Several types of bezoars exist[1], Uncluding phytobezoar(plant materiel), lactobezoar (milk seen exclusively in infants),pharmacobezoar (medications)and trichobezoard(hair).Bezoar are Most commonly seen in the stomach[2], and may Also involve the small bowel particulary in the case Of trichobezoars[3]. Trichobezoars overwhelmingly tend to occur in females over males, with predisposing factors of trichotillomania and trichophagia[4]. Common presenting signs and symptoms of trichobezoars include chronic gastrointestinal, a palpable abdominal mass, and small bowel obstruction. The dreded complication of trichob-ezouars is stomach or bowel perforation [5]. Imaging plays an important role in diagnosis.on abdom-inal radiography, the diagnosis may be suspected when there is abundant mottled material admixed with gas distending the stomach [6]. On CT, bezoars present as a heterogeneous intraluminal mass typically intersper- ed with gas, distending but not adherent to the stom-ach or small bowel. CT is helpful in delineating small small bowel extension including more distal satellite lesion [7].

\section{CONCLUSION}

In summary, our case highlights trichobezoar as an Uncommen, yet important potential cause of abdom Inal pain in girls presenting with acute abdominal Pain .The diagnosis of trichobezoar may be suspected In female patients with the history of trichotillomania, Trichophagia,gastrointestinal symptoms and a palpable Abdominal mass. However imaging is a mainstay for accurate diagnosis particulary of potential complications.

\section{Abbreviations}

CT: Computed tomography.

\section{ACKNOWLEDGEMENTS}

We thank the patient for giving his informed consent to publish this case report.

\section{REFFERENCE}

1. Lynch KA, Feola PG, Guenther E. Gastric trichobezoar: an important cause of abdominal pain presenting to the pediatric emergency department. Pediatr Emerg Care. 2003;19(5):343347.

2. Otjen JP, Iyer RS, Phillips GS, Parisi MT. Usual and unusual causes of pediatric gastric outlet obstruction. Pediatr Radiol. 2012;42(6):728-737.

3. Naik S, Gupta V, Naik S, Rangole A, Chaudhary AK, Jain P. Rapunzel syndrome reviewed and redefined. Dig Surg. 2007;24(3):157-161.

4. Gorter RR, Kneepkens CM, Mattens EC, Aronson DC, Heij HA. Management of trichobezoar: case report and literature review. Pediatr Surg Int. 2010;26(5):457-463.

5. Fallon SC, Slater BJ, Larimer EL, Brandt ML, Lopez ME. The surgical management of Rapunzel syndrome: a case series and literature review. J Pediatr Surg. 2013;48(4):830-834.

6. Gayer G, Jonas T, Apter S, Zissin R, Katz M, Katz R. Bezoars in the stomach and small bowel-CT appearance. Clin Radiol. 1999;54(4):228-232.

7. Dalshaug GB, Wainer S, Hollaar GL. The Rapunzel syndrome (trichobezoar) causing atypical intussusception in a child: a case report. J Pediatr Surg. 1999;34(3):479-480. 